\title{
1 Monitoring migratory birds in stopover habitat: 2 assessing the value of extended duration audio 3 recording
}

5 Running title: Monitor migratory birds with audio recording

7 Ellie Roark*1, Willson Gaul ${ }^{2}$

$8 \quad{ }^{1}$ Dublin, Ireland

$9 \quad{ }^{2}$ School of Biology and Environmental Science, Earth Institute, University College Dublin, Ireland

$10 *$ Corresponding author: ellieroark@gmail.com

\section{ABSTRACT}

1. Because birds are frequently detected by sound, autonomous audio recorders (called automated recording units or ARUs) are now an established tool in addition to in-person observations for monitoring the status and trends of bird populations. ARUs have been evaluated and applied during breeding seasons, and to monitor the nocturnal flight calls of migrating birds. However, birds behave differently during migration stopover than during the breeding season. Here we present a method for using ARUs to monitor land birds in migration stopover habitat.

2. We conducted in-person point counts next to continuously recording ARUs, and compared estimates of the number of species detected and focal species relative abundance from point counts and ARUs. We used a desk-based audio bird survey method for processing audio recordings, which does not require automated species identification algorithms. We tested two 
methods of using extended duration ARU recording: surveying consecutive minutes, and surveying randomly selected minutes.

3. Desk-based surveys using randomly selected minutes from extended duration ARU recordings performed similarly to point counts, and better than desk-based surveys using consecutive minutes from ARU recordings. Surveying randomly selected minutes from ARUs provided estimates of relative abundance that were strongly correlated with estimates from point counts, and successfully showed the increase in abundance associated with migration timing. Randomly selected minutes also provided estimates of the number of species present that were comparable to estimates from point counts.

4. ARUs are an effective way to track migration timing and intensity in remote or seasonally inaccessible migration stopover habitats. We recommend that desk-based surveys use randomly sampled minutes from extended duration ARU recordings, rather than using consecutive minutes from recordings. Our methods can be immediately applied by researchers with the skills to conduct point counts, with no additional expertise necessary in automated species identification algorithms.

\section{Keywords}

autonomous audio recording, bird survey, migration, stopover habitat, passive acoustic monitoring, point count, relative abundance 


\section{1 | INTRODUCTION}

Conserving bird populations requires knowledge of bird distribution and habitat use at all stages of their life cycle, including during breeding, migration, and non-breeding periods (Sherry \& Holmes, 1995) . Monitoring birds' habitat use during migration is a necessary component of conservation plans for migratory birds. Historically, researchers have primarily relied on in-person observations including mist-netting (Peach, Buckland \& Baillie, 1996) and point counts (Ralph, Droege \& Sauer, 1995) for migration monitoring, but because birds are frequently detected by sound, audio recording technology offers opportunities to expand monitoring techniques. Here we present a method for using audio recorders to monitor birds in migration stopover habitat during spring migration.

Figuring out how to best monitor bird abundance and diversity in remote habitat is a current challenge. The climate in high latitude continental regions increases the challenges associated with accessing remote areas during spring migration. Significant annual snow accumulation, followed by rapid melting as temperature increases, makes unpaved roads impassable for several weeks each spring in much of northern North America, typically during the same time period when migrant bird species begin to arrive in the region. Developing survey monitoring protocols that can be implemented despite poor traveling conditions is a way to fill in gaps in knowledge of northern forest birds, and birds in similarly remote habitats.

Autonomous recording units (ARUs) are programmable audio recorders that can be deployed in the field for long time periods to efficiently maximize the spatial and temporal extent of monitoring. Passive acoustic monitoring is widely used in ecology to monitor and study vocalizing organisms; ARUs have been deployed to study bats (Tuneu-Corral et al., 2020), whales (Baumgartner et al., 2019), invertebrates (Penone et al., 2013), amphibians (Dutilleux \& Curé, 2020) 
and birds (Shonfield \& Bayne, 2017). ARUs are also used to evaluate the success of conservation programs (Shonfield \& Bayne, 2017). Current challenges for implementing passive acoustic monitoring include the availability of reference sound libraries, minimizing errors in species identification, and determining the relationship between acoustic index values and their associated realworld underlying parameters (Gibb, Browning, Glover-Kapfer \& Jones, 2019).

Point-count surveys are the most commonly used bird monitoring protocol for long-term study sites (Ralph, Droege, \& Sauer, 1995; Rosenstock, Anderson, Giesen, Leukering \& Carter, 2002), but ARUs are now viewed as a viable supplement to point-counts, especially during the breeding season when birds vocalize frequently (Furnas \& Callas, 2015; Klingbeil \& Willig, 2015; Shonfield \& Bayne, 2017; Darras et al., 2018; Darras et al., 2019). Many researchers have compared ARUs and point counts in terms of their estimates of species richness and relative abundance or occupancy (Haselmayer \& Quinn, 2000; Campbell \& Francis, 2011; Tegeler, Morrison \& Szewczak, 2012; La \& Nudds, 2016), including in temperate forest (Klingbeil \& Willig, 2015). However, none of these studies (including the 23 studies reviewed in Darras et al.’s (2018) meta-analysis) compared point counts and ARUs during migration. Birds behave and vocalize differently during migration than during the breeding season (Morse, 1991; Rappole \& Warner, 1976). Testing and refining migration-specific monitoring techniques for ARUs is therefore necessary to understand how data from ARUs compare to data from in-person observations.

ARUs are currently used during migration to record the flight calls of nocturnally migrating species. They are deployed to track the abundance of migrants as they move through an area, and can provide helpful information about migratory flyway locations, migration phenology, and relative abundance (Sanders \& Mennill, 2014; Evans \& Rosenburg, 2000). Understanding how migrating birds use migratory stopover habitat is a different challenge, and requires different methods. Determining 
how birds are distributed in stopover habitat, the relative abundance and species richness of birds in such habitat, and the timing of arrival and departure from the stopover area are all important research questions for applied conservation.

To take advantage of the large volume of data generated by continuously recording ARUs, researchers are actively developing methods for automated identification of vocalizing organisms (Salamon et al., 2016; Gibb, Browning, Glover-Kapfer \& Jones, 2019). In contrast, we present a method that can be implemented by anyone with the skills to conduct point counts, that does not rely on machine learning for species identification and data processing. Because applications of ARUs in migration stopover habitat have been under-explored in the literature thus far, we demonstrated and assessed an immediately applicable monitoring technique.

We compared data from ARU surveys to in-person point count surveys during spring migration in the northern Great Lakes region of the United States. Our goal was to understand how ARUs could be applied to monitor diurnal stopover habitat use during migration by examining whether ARUs could provide estimates of relative abundance and number of species that are comparable to estimates from in-person surveys. We asked the following questions. 1) What are the differences between the number of species detected using point counts and using ARUs? 2) Can ARUs give estimates of relative abundance for focal species that are correlated with estimates of relative abundance from point counts?

3) Can randomly sampling from extended duration audio recordings provide better estimates of focal species abundance or the number of species detected than consecutive minutes of audio recording? 


\section{2 | MATERIALS AND METHODS}

We conducted in-person point counts alongside continuously recording ARUs on the southern shore of Lake Superior during two months at the start of spring migration. We compared both raw data and model-based estimates of the number of species detected and focal species abundance from point counts and ARUs.

\subsection{Study site}

We conducted field work in a $2.7 \mathrm{~km}^{2}$ area on the Point Abbaye peninsula in Baraga County, Michigan, USA (Fig. 1). Surveys took place from 2 April to 22 May 2019, and were conducted daily unless prevented by weather conditions. Field work was designed to coincide with the arrival and peak abundance of early season migrating birds. Point Abbaye juts into the southern part of Lake Superior and comprises the western border of Keweenaw Bay. Habitat included forested wetland, upland hardwood, and hardwood forest disturbed by recent logging activity. We selected survey sites randomly across the study area. All spatial analyses were done in the R programming language using the 'rgdal', 'geosphere', 'rgeos', 'sp', 'maptools', and 'spatstat' packages (Baddeley, Rubak, \& Turner, 2015; Bivand, Keitt, \& Rowlingson, 2018; Bivand \& Lewin-Koh, 2019; Bivand \& Rundel, 2018; Bivand, Pebesma, \& Gomez-Rubio, 2013; Hijmans, 2019; Pebesma \& Bivand, 2005; R Core Team, 2020). We conducted a pilot study in 2018 to test our protocols and evaluate the accessibility of our randomly selected survey locations. See Appendix A for details about pilot year surveys, and survey site and date selection.

\section{2 | Automated recording units}

Birds were recorded using three SWIFT bioacoustic recorder rugged units (Cornell Lab of Ornithology, Ithaca, NY, USA), and one AudioMoth bioacoustic recorder that was housed in a thin plastic bag for light weather proofing (Hill et al., 2018; Open Acoustic Devices, Southampton, UK). SWIFT units used a built in PUI Audio brand omni-directional microphone. The AudioMoth unit used 
131 an analog microelectro-mechanical systems (MEMS) microphone. We refer to both the SWIFT and

132 AudioMoth units as “automated recording units” (ARUs). ARUs recorded at a sampling rate of $48 \mathrm{kHz}$

133 and saved recordings as uncompressed .WAV files. The signal to noise ratio reported by device

134 manufacturers is approximately $58 \mathrm{~dB}$ for the SWIFT units, and approximately $44 \mathrm{~dB}$ for the

135 AudioMoth unit.

136

137

138

139

140

141

142

143

144

145

146

147

148

149

150

151

152

\section{4 | Field survey methods}

ARUs recorded continuously for five hours each day, beginning within 10 minutes of local sunrise time (United States Naval Observatory, 2016). ARUs were attached to trees less than $0.6 \mathrm{~m}$ in diameter, and were placed 1.5-2 m above the ground (Darras et al., 2018). The SWIFT omnidirectional microphones were always oriented downward to prevent precipitation landing directly on the microphone. After the five hour recording period ended each day, ARUs were moved to new locations for the next day's samples, thereby rotating the ARU and point count samples through all 18 survey locations approximately every five days. The sampling order for the points was chosen randomly.

Point counts were conducted daily next to each ARU during the five hour recording period.

Point counts involved recording all birds seen and heard at an unlimited distance during a stationary, 10-minute count. We did not survey in high wind or heavy precipitation. See Appendix A for detailed point count protocols.

\section{5 | Desk-based audio surveys}

We conducted desk-based audio bird surveys by listening to ARU recordings played through headphones on a laptop computer in the lab after the end of the field season. We tested three types of desk-based audio surveys: 1) we listened to a recording of the 10 consecutive minutes during which the 
in-person point count was conducted; 2) we listened to 24 minutes selected randomly from the five hour recording duration; 3) we sampled a subset of 10 of the 24 random minutes (without listening to those minutes again). Our goal was to compare each of these desk-based ARU survey methods to inperson point count observations.

For each audio file, the desk-based survey technician noted the identity of each bird species that vocalized, the type of vocalization, and the 30-second time intervals in which each species vocalized. Detailed protocols for completing desk-based audio surveys can be found in Appendix A, and a completed data sheet from a desk-based survey is shown in Fig. S6. We sampled 24 random minutes from each ARU on each day because we wanted at least 20 minutes without anthropogenic disturbance. After discarding randomly selected minutes that contained a human voice, we were ultimately able to use data from 22 randomly selected minutes from each ARU on each survey day (i.e. we never had to discard more than two of the 24 randomly selected minutes because of human voices).

\subsection{Indices for observed number of species and relative abundance}

We summed the number of unique species detected $(S)$ separately using each survey type: 10minute, in-person point counts $\left(S_{\mathrm{p}}\right)$; 10 consecutive minute ARU surveys $\left(S_{10 \mathrm{c}}\right)$; 10 random minute ARU surveys $\left(S_{10 \mathrm{R}}\right)$; and 22 random minute ARU surveys $\left(S_{22 \mathrm{R}}\right)$ (Box 1). We calculated a value of $S$ for each individual survey on each day, resulting in three or four values of $S$ for each survey type on each day.

We created an index of daily relative abundance $(A)$ for two focal species (Regulus satrapa (Golden-crowned Kinglet) and Troglodytes hiemalis (Winter Wren)) using each survey type (Box 1). Our relative abundance indices were: the mean observed number of individuals per point count $\left(A_{\mathrm{p}}\right)$; the proportion of 30-second intervals with a vocalization calculated by surveying $n$ minutes in sections 
of 10 consecutive minutes $\left(A_{n c}\right)$; the proportion of 30-second intervals with a vocalization calculated by surveying $n$ minutes in sections of one minute chosen randomly from the five hour survey window $\left(A_{n \mathrm{R}}\right)$. To reduce the number of zero abundance counts in our data, we calculated relative abundance indices by grouping all surveys of each type for each day, so there was a single value for each abundance index on each day. Note that while April $16^{\text {th }}$ and $17^{\text {th }}$ data appear on plots and in results, ARU malfunctions on those dates made the number of sampled minutes $n$ different for those two dates for some of our abundance indices. See Appendix A for detailed discussion of sample size on these dates.

\section{7 | Statistical Analysis}

\subsection{1 | Observed number of species}

To determine whether the survey type $\left(S_{10 \mathrm{C}}, S_{10 \mathrm{R}}, S_{22 \mathrm{R}}\right)$ significantly influenced the number of species detected, we modeled the number of species detected using a generalized linear mixed model (GLMM) with a Poisson error distribution and log link function, using the 'Ime4' package in R (Bates, Maechler, Bolker, \& Walker, 2015; R Core Team, 2020). Our fixed effects were survey type, day of year, a second degree polynomial term for day of year, wind, rain, noise, and interaction terms for day of year x survey type, and survey type x rain. We also used day of year as a random effect; we collected up to four samples of each of four survey types per day, and new birds potentially arrived daily during the study period, so we expected that the number of species detected by all surveys on each day would be strongly correlated, regardless of survey location or survey type. More information about our GLMM can be found in Appendix A.

\subsection{2 | Relative abundance}

We compared relative abundance estimates for our two focal species using data from $A_{\mathrm{p}}$ and from each of the three desk-based audio survey types $\left(A_{30 \mathrm{c}}, A_{30 \mathrm{R}}, A_{66 \mathrm{R}}\right)$. Winter Wrens were abundant in 
198 the survey area, and vocalized frequently and loudly during early spring, representing a "best case”

199 scenario for detectability on ARU recordings. Golden-crowned Kinglets were abundant in the survey

200 area, but vocalized quietly (though regularly) during early spring, and so represent a greater challenge

201 for detection using ARUs.

We produced a total of eight relative abundance models, one using each of our four relative

abundance indices for each of our two focal species. For each relative abundance model, we fit

iterations of five-fold temporal block cross validation, that used blocks of three consecutive days (Fig.

S3; Roberts et al., 2017). This resulted in a total of 1000 BRT fits per relative abundance model. We

210 (Greenwell, Boehmke, Cunningham and GBM Developers, 2019; R Core Team, 2020). Details of

\section{3 | RESULTS}


that prevented us from recording the full five-hour survey window with some units on some days. One

SWIFT unit recorded 36 five-hour survey days, two SWIFT units recorded 35 five-hour survey days, and the AudioMoth unit recorded 24 five-hour survey days, for a total of 650 hours recorded by ARUs. Because of ARU malfunctions, on some days ARUs did not record the full five-hour period, but we were able to manually turn on the units for the 10-minute period during the in-person point count.

Therefore, we recorded 13010 consecutive minute periods with ARUs (during which a human observer was present conducting a simultaneous point count) on 37 survey days, but only 124 periods of 22 randomly selected minutes on 36 survey days. A complete list of the species detected by each survey method can be found in Table S1.

\section{1 | Observed number of species}

A chi-square ANOVA comparing our full model to a null model with survey type removed showed that survey type (the $S$-index used) had a significant effect on the number of species detected $\left(X_{9,21}^{2}=247, p<0.0001\right)$. We detected a similar number of species using $S_{10 \mathrm{R}}$ as we did using $S_{\mathrm{p}}$ (Fig. 2;

Table 1; change in the log of the number of species detected $=-0.065,95 \%$ CI $[-0.2 ; 0.08], p=0.3888$ ). of the number of species detected $=0.305,95 \%$ CI $[0.17 ; 0.44], p<0.0001)$. We detected fewer species using $S_{10 \mathrm{c}}$ than using $S_{\mathrm{p}}$ (Fig. 2; Table 1; change in the log of the number of species detected $=-0.614$, 95\% CI [-0.78; -0.44], $p<0.0001)$. Listening to randomly selected rather than consecutive minutes eliminated the gap in number of species detected between 10-minute point counts and 10-minute ARU more species expected later in the migration season (Fig. 2). 
Chi-square ANOVA showed that the overall effect of wind was not significant $\left(\chi^{2}{ }_{19,21}=1.44, p=\right.$

observed number of species over the course of the survey season.

\section{2 | Relative abundance models}

BRT models of relative abundance over time differed in how well they showed the initial period of absence, and the increase in abundance corresponding with the arrival of migrant birds in our study area, depending on the survey method used (Fig. 3). The general pattern of initial absence followed by arrival of migrants can be seen in both the raw data and the model predictions of relative abundance for $A_{\mathrm{p}}, A_{30 \mathrm{R}}$ and $A_{66 \mathrm{R}}$ for Winter Wrens (Fig. 3 a, c and d), and for $A_{\mathrm{p}}$ and $A_{66 \mathrm{R}}$ for Golden-crowned Kinglets (Fig. 3 e, and h).

For both Winter Wrens and Golden-crowned Kinglets, the observed abundance indices from ARU surveys were positively correlated with the observed abundance index from point counts (Fig. 4, Fig. 5), indicating that the relative abundance proxies we calculated using ARUs are comparable to relative abundance estimates from in-person observations. Winter Wren showed moderate to strong correlation between the observed abundance index values from point counts and from ARU surveys (Fig. 4, Table 2). Abundance indices for Golden-crowned Kinglets were less correlated than for Winter Wrens, with weak correlation between $A_{30 \mathrm{C}}$ and $A_{30 \mathrm{R}}$ in particular (Fig. 5, Table 2). Correlations for predicted values of our abundance indices were moderate to strong for both species (Figs. S1-S2), and were higher than correlation coefficients for observed values of the same index pairs (Table 2). The 
strong correlation between predicted values of the abundance indices indicates that our models found the same underlying signal regardless of whether training data were from ARUs or point counts.

For Winter Wrens, observed abundance indices using randomly selected minutes from ARUs $\left(A_{30 \mathrm{R}}, A_{66 \mathrm{R}}\right)$ were more closely correlated with the observed abundance index from point counts $\left(A_{\mathrm{P}}\right)$ than was the abundance index from 10 consecutive minute ARU surveys $\left(A_{30 \mathrm{C}}\right)$. For both species, $A_{\mathrm{P}}$ was most strongly correlated with $A_{66 \mathrm{R}}$.

\section{4 | DISCUSSION}

Importantly, abundance models trained with ARU data showed the increase in relative abundance indicating the arrival of migrants at the study site, suggesting that ARUs can be used to track migration phenology in stopover habitat for vocal species. ARUs also provided similar estimates of the number of species detected as point counts when analyzing randomly sampled minutes. Our results suggest that ARUs recording for an extended duration in migration stopover habitat can be just as effective as in-person point counts for monitoring migrating land birds.

Data from randomly selected minutes of ARU recordings detected more species and produced modeled abundance estimates that better showed the expected seasonal pattern of migration timing than data from consecutive minutes of ARU recordings. There are two likely explanations for this. First, randomly selected minutes are less temporally auto-correlated than consecutive minutes. For example, during a 10-minute in-person point count, little new information is gained during the seventh minute of the survey compared to what was collected during the sixth minute of the survey; a Winter Wren singing near the end of the sixth minute of a point count survey will likely still be singing in the beginning of the seventh minute. By selecting minutes randomly from across the five-hour survey window, the temporal correlation between each successive minute that is analyzed is minimized. 
Second, during migration stopover, birds may move more and farther distances within the study area than they would during the breeding season, when they have established a territory. The community of birds within the immediate detection radius of an observer (either a person or a recording ARU) may therefore change over the course of five hours. Using randomly selected minutes provides a more complete sample of the birds using a spatial location over the entire course of the survey window. For in-person point counts, the time taken to travel to a survey site takes up a major portion of the total time invested, so site visits are typically limited to once per day. With ARUs, no such constraints exist; it is possible to do multiple short-duration surveys from many locations over the course of one day without additional travel and field work logistics. We recommend that studies using ARUs on migration should randomly sample recordings of short periods of time (e.g. one-minute recordings) from a defined survey window relevant to the study question (e.g. the five hours following sunrise for passerines in temperate forest, or twilight to dawn for crepuscular and nocturnal species). In many studies using ARUs during the breeding season, researchers listened to consecutive minutes of recordings (e.g. 10 minutes or 2 minutes in Klingbeil \& Willig (2015)). Given the improvement we saw when listening to random rather than consecutive minutes from an extended duration recording, we recommend that future studies using ARUs to monitor birds during wintering or breeding seasons test whether randomly selected minutes provide a more effective sample than consecutive minutes. We did not detect an effect of either wind or rain in our model of the number of species detected. However, because we controlled for adverse weather conditions during our field surveys by not surveying on rainy or windy days, the number of high wind values in our data was low, as was the number of rainy survey days. We also noted anecdotally that occasionally the wind values recorded in person for a survey day did not correlate with the amount of wind heard while conducting our deskbased audio surveys; we speculate that wind direction in relation to the microphone may make a 
difference in how much wind is actually picked up by the ARU. Given that wind and rain have an

effect on the detectability of birds in the study system (Ralph, Droege \& Sauer, 1995), they remain important predictors to include, whether or not they appear significant in our model. Likewise, we suspect that the insignificance of the polynomial day of year term is due to sample size limitations. Interpreting the significance of the noise variable is challenging, because we used the variable to describe all non-avian noise in the environment, which could include waves, airplanes, and frogs. We suspect that the overall significance of the noise variable may be due to frogs. Future studies may want to carefully consider whether they wish to distinguish between other vocalizing taxa and surrounding environmental noise. ARUs can be used to simultaneously sample multiple taxa (e.g. crickets and bats; Newson, Bas, Murray \& Gillings, 2017) so researchers may want to incorporate analyses of non-bird biotic noise into study designs.

Estimates of abundance are more useful than estimates of occupancy for prioritizing conservation resources at dynamic temporal scales, such as during migration (Johnston et al., 2015). ARUs do not solve the problem of how to estimate true abundance in stopover habitat during migration. Imperfect detection means that the number of individuals detected is not necessarily a good estimate of the number of individuals present (MacKenzie \& Kendall, 2002). Hierarchical models that account for imperfect detection (MacKenzie et al., 2002; Kéry \& Royle, 2016) rely on assumptions about population closure that may be badly violated during migration, when birds do not adhere to territories and are present in stopover habitat for short periods of time. The period in which we can reasonably assume population closure for our study area during migration may be as short as several hours or as long as several days, depending on weather conditions. Therefore disentangling true occupancy or abundance from detectability is difficult, whether using traditional in-person survey methods or ARUs. Using a relative abundance index that does not account for detection probability 
could result in misleading estimates of abundance if detection probability is not constant (MacKenzie

331

332

333

\& Kendall, 2002). It is possible that individual birds’ vocalizations may increase over the spring migration period, as birds prepare for the breeding season. Using our abundance indices, increases in vocalizations would look like an increase in relative abundance, but the apparent increase would merely be an artifact of changing detectability. However, given the moderate (for Golden-crowned Kinglets) and strong (for Winter Wrens) correlation between $A_{\mathrm{p}}$ (observed abundance from point counts) and our ARU abundance indices, we believe increases in relative abundance seen in our model results (Fig. 3) are not mere artifacts of changes in detectability, but rather show real increases in abundance associated with the arrival of our focal species in the study area. ARUs can therefore provide valuable data about migration phenology and stopover habitat use, even if they cannot be used to estimate true abundance.

Differences in how well relative abundance models captured the arrival of migrants seemed to be partly dictated by how well aggregating detections by day reduced the number of "zero" counts in our data. For Golden-crowned Kinglets, the $A_{\mathrm{p}}$ and $A_{66 \mathrm{R}}$ models seemed to be effective at detecting the increase in abundance associated with initial arrival, though they did not capture a peak of abundance, if one existed during the study period. It is unclear whether days with high counts of Golden-crowned Kinglets represent a real pulse of newly arrived birds, or the same birds already present in the region clustering more densely, or just random variation around a more or less constant number of individuals. A dense network of simultaneously recording ARUs in the region could help answer this question. Future studies might also consider increasing ARU survey effort beyond our maximum of 66 randomly selected minutes per day. The improvement in our models associated with increasing from 30 to 66 minutes suggests that increasing the number of minutes surveyed is beneficial. 

period as birds approach their breeding grounds. access migratory stopover habitat in high latitude forests. Temporal variation in accessibility in these habitats is dramatic, as unpaved roads typically turn from snow to slush to impassable mud before hardening into reliably dry surfaces in early summer. ARUs can eliminate many of the restrictive logistics and safety concerns for researchers interested in monitoring spring migration. Our method of the skills to conduct point counts. Researchers can set up ARUs during winter conditions when access collect the audio data once conditions have stabilized in late spring. Our methods for using ARU data to model relative abundance of focal species and the number of species present during migration can be immediately applied to increase monitoring effort in logistically difficult regions.

\section{Acknowledgments}

This work was funded by grants to ER from the Wilson Ornithological Society and the Copper Country 
Dana Neufield for assistance with the pilot year surveys, and to Jon Yearsley, Hannah White, and the Ecological Modeling lab at University College Dublin for consultation on modeling methods. WG was funded during this work by Science Foundation Ireland grant number 15/IA/2881.

\section{Author Contributions}

ER and WG developed the study design and methodology; ER prepared for and performed all field work, and conducted all desk-based audio surveys; ER and WG conducted the data analysis, wrote the manuscript, and gave final approval for publication.

\section{Data Availability Statement}

Data and code to reproduce analyses can be found at https://doi.org/10.5281/zenodo.3964500

(ellieroark, 2020). Audio recording files used to produce this analysis are archived at

https://doi.org/10.5281/zenodo.3964574 (Roark \& Gaul, 2020).

\section{References}

Baddeley, A., Rubak, E., \& Turner, R. (2015). Spatial Point Patterns: Methodology and Applications with R. Retrieved from http://www.crcpress.com/Spatial-Point-Patterns-Methodology-andApplications-with-R/Baddeley-Rubak-Turner/9781482210200/

Bates, D., Maechler, M., Bolker, B., \& Walker, S. (2015). Fitting Linear Mixed-Effects Models Using lme4. Journal of Statistical Software, 67(1), 1-48. https://doi.org/10.18637/jss.v067.i01

Baumgartner, M. F., Bonnell, J., Van Parijs, S. M., Corkeron, P. J., Hotchkin, C., Ball, K., ... Kraus, S. D. (2019). Persistent near real-time passive acoustic monitoring for baleen whales from a moored buoy: System description and evaluation. Methods in Ecology and Evolution, 10(9), 1476-1489. https://doi.org/10.1111/2041-210X.13244

Beaufort, F. (1805). Beaufort Wind Scale. Retrieved July 28, 2020, from NOAA.gov website: https://www.spc.noaa.gov/faq/tornado/beaufort.html

Bivand, R., Keitt, T., \& Rowlingson, B. (2018). rgdal: Bindings for the “Geospatial” Data Abstraction Library. Retrieved from https://cran.r-project.org/package=rgdal

Bivand, R., \& Lewin-Koh, N. (2019). maptools: Tools for Handling Spatial Objects. Retrieved from https://cran.r-project.org/package=maptools 
Bivand, R., \& Rundel, C. (2018). rgeos: Interface to Geometry Engine- Open Source ('GEOS'). Retrieved from https://cran.r-project.org/package=rgeos

Bivand, R. S., Pebesma, E. J., \& Gomez-Rubio, V. (2013). Applied spatial data analysis with R (2nd ed.). Retrieved from http://www.asdar-book.org/\%0A

Campbell, M., \& Francis, C. M. (2011). Using Stereo-Microphones to Evaluate Observer Variation In North American Breeding Bird Survey Point Counts. The Auk, 128(2), 303-312. https://doi.org/10.1525/auk.2011.10005

Darras, K., Batáry, P., Furnas, B., Celis-Murillo, A., Van Wilgenburg, S. L., Mulyani, Y. A., \& Tscharntke, T. (2018). Comparing the sampling performance of sound recorders versus point counts in bird surveys: A meta-analysis. Journal of Applied Ecology, 55(6), 2575-2586. https://doi.org/10.1111/1365-2664.13229

Darras, K., Batáry, P., Furnas, B. J., Grass, I., Mulyani, Y. A., \& Tscharntke, T. (2019). Autonomous sound recording outperforms human observation for sampling birds: a systematic map and user guide. Ecological Applications, 29(6). https://doi.org/10.1002/eap.1954

Dutilleux, G., \& Curé, C. (2020). Automated acoustic monitoring of endangered common spadefoot toad populations reveals patterns of vocal activity. Freshwater Biology, 65(1), 20-36. https://doi.org/10.1111/fwb.13111

Elith, J., Leathwick, J. R., \& Hastie, T. (2008). A working guide to boosted regression trees. Journal of Animal Ecology, 77(4), 802-813. https://doi.org/10.1111/j.1365-2656.2008.01390.x

ellieroark. (2020, July 28). ellieroark/StopoverHabitatMonitoring: First release of Stopover Habitat Monitoring project code (Version v1.0.0). Zenodo. http://doi.org/10.5281/zenodo.3964500

Evans, W. R., \& Rosenberg, K. V. (2000). Acoustic Monitoring of Night-Migrating Birds : A Progress Report. In: Strategies for bird conservation: creating the Partners in Flight planning process. Proceedings of the 3rd Partners in Flight Workshop. US Department of Agriculture, Forest Service.

Friedman, J. (2001). Greedy Function Approximation : A Gradient Boosting Machine. The Annals of Statistics, 29(5), 1189-1232. https://doi.org/10.1214/009053606000000795

Furnas, B. J., \& Callas, R. L. (2015). Using automated recorders and occupancy models to monitor common forest birds across a large geographic region. Journal of Wildlife Management, 79(2), 325-337. https://doi.org/10.1002/jwmg.821

Gibb, R., Browning, E., Glover-Kapfer, P., \& Jones, K. E. (2019). Emerging opportunities and challenges for passive acoustics in ecological assessment and monitoring. Methods Ecol Evol, 10, 169-185. https://doi.org/10.1111/2041-210X.13101 
Greenwell, B., Boehmke, B., Cunningham, J., \& GBM Developers. (2019). gbm: Generalized Boosted Regression Models. R package version 2.1.5. R package version 2.1.5.

Haselmayer, J., \& Quinn, J. S. (2000). A Comparison of Point Counts and Sound Recording as Bird Survey Methods in Amazonian Southeast Peru. The Condor, 102(4), 887-893. Retrieved from http://www.jstor.org/stable/1370317

Hijmans, R. J. (2019). geosphere: Spherical Trigonometry. Retrieved from https://cran.r-project.org/package=geosphere

Hill, A. P., Prince, P., Piña Covarrubias, E., Doncaster, C. P., Snaddon, J. L., \& Rogers, A. (2018). AudioMoth: Evaluation of a smart open acoustic device for monitoring biodiversity and the environment. Methods in Ecology and Evolution, 9(5). https://doi.org/10.1111/2041-210X.12955

Johnston, A., Fink, D., Reynolds, M. D., Hochachka, W. M., Sullivan, B. L., Bruns, N. E., ... Kelling, S. (2015). Abundance models improve spatial and temporal prioritization of conservation resources. Ecological Applications, 25(7), 1749-1756. https://doi.org/10.1890/07-1650.1

Kéry, M., \& Royle, J. A. (2016). Applied hierarchical modeling in ecology: analysis of distribution, abundance and species richness in R and BUGS (volume 1 - prelude and static models). London: Academic Press.

Klingbeil, B. T., \& Willig, M. R. (2015). Bird biodiversity assessments in temperate forest: the value of point count versus acoustic monitoring protocols. PeerJ, 3, e973. https://doi.org/10.7717/peerj.973

La, V. T., \& Nudds, T. D. (2016). Estimation of avian species richness: biases in morning surveys and efficient sampling from acoustic recordings. Ecosphere, 7(4), e01294. https://doi.org/10.1002/ecs2.1294

MacKenzie, D. I., \& Kendall, W. C. (2002). How should detection probability be incorporated into estimates of relative abundance? Ecology, 83(9), 2387-2393. https://doi.org/10.1890/00129658(2002)083[2387:HSDPBI]2.0.CO;2

MacKenzie, D. I., Nichols, J. D., Lachman, G. B., Droege, S., Royle, A. A., \& Langtimm, C. A. (2002). Estimating site occupancy rates when detection probabilities are less than one. Ecology, 83(8), 2248-2255. https://doi.org/10.1890/0012-9658(2002)083[2248:ESORWD]2.0.CO;2

Morse, D. H. . (1991). Song Types of Black-Throated Green Warblers on Migration. The Wilson Bulletin, 103(1), 93-96.

Newson, S. E., Bas, Y., Murray, A., \& Gillings, S. (2017). Potential for coupling the monitoring of bush-crickets with established large-scale acoustic monitoring of bats. Methods in Ecology and Evolution, 8(9), 1051-1062. https://doi.org/10.1111/2041-210X.12720 
Peach, W. J., Buckland, S. T., \& Baillie, S. R. (1996). The use of constant effort mist-netting to measure between-year changes in the abundance and productivity of common passerines. Bird Study, 43(2), 142-156. https://doi.org/10.1080/00063659609461007

Pebesma, E. J., \& Bivand, R. (2005). Classes and methods for spatial data in R. Retrieved from R News 5 (2) website: ttps://cran.r-project.org/doc/Rnews/

Penone, C., Le Viol, I., Pellissier, V., Julien, J. F., Bas, Y., \& Kerbiriou, C. (2013). Use of large-scale acoustic monitoring to assess anthropogenic pressures on orthoptera communities. Conservation Biology, 27(5), 979-987. https://doi.org/10.1111/cobi.12083

R Core Team. (2020). R: A Language and Environment for Statistical Computing. Retrieved from https://www.r-project.org/

Ralph, C. J., Droege, S., \& Sauer, J. R. (1995). Managing and Monitoring Birds Using Point Counts: Standards and Applications. Workshop on Monitoring Bird Population Trends by Point Counts, 161-169.

Rappole, J. H., \& Warner, D. W. (1976). Relationships between Behavior, Physiology and Weather in Avian Transients at a Migration Stopover Site. Oecologia, (26), 193-212.

Roark, E., \& Gaul, W. (2020). Point Abbaye Stopover Habitat Monitoring Audio Files [Data set]. Zenodo. http://doi.org/10.5281/zenodo.3964574

Roberts, D. R., Bahn, V., Ciuti, S., Boyce, M. S., Elith, J., Guillera-Arroita, G., ... Dormann, C. F. (2017). Cross-validation strategies for data with temporal, spatial, hierarchical, or phylogenetic structure. Ecography, 40, 913-929. https://doi.org/10.1111/ecog.02881

Rosenstock, S. S., Anderson, D. R., Giesen, K. M., Leukering, T., \& Carter, M. F. (2002). Landbird Counting Techniques : Current Practices and an Alternative Published. The Auk, 119(1), 46-53. https://www.jstor.org/stable/4090011

Salamon, J., Bello, J. P., Farnsworth, A., Robbins, M., Keen, S., Klinck, H., \& Kelling, S. (2016). Towards the Automatic Classification of Avian Flight Calls for Bioacoustic Monitoring. PloS ONE, 11(11). https://doi.org/10.1371/journal.pone.0166866

Sanders, C. E., \& Mennill, D. J. (2014). Acoustic monitoring of nocturnally migrating birds accurately assesses the timing and magnitude of migration through the Great Lakes. The Condor, 116(3), 371-383. https://doi.org/10.1650/condor-13-098.1

Sherry, T. W., \& Holmes, R. T. (1995). Summer versus winter limitation of populations: What are the issues and what is the evidence? In T. E. Martin \& D. M. Finch (Eds.), Ecology and management of Neotropical migratory birds: A synthesis and review of critical issues (pp. 85-120). 
Shonfield, J., \& Bayne, E. M. (2017). Autonomous recording units in avian ecological research: current use and future applications. Avian Conservation and Ecology, 12(1). https://doi.org/10.5751/ace00974-120114

Tegeler, A. K., Morrison, M. L., \& Szewczak, J. M. (2012). Using extended-duration audio recordings to survey avian species. Wildlife Society Bulletin, 36(1), 21-29. https://doi.org/10.1002/wsb.112

Tuneu-Corral, C., Puig-Montserrat, X., Flaquer, C., Mas, M., Budinski, I., \& López-Baucells, A. (2020). Ecological indices in long-term acoustic bat surveys for assessing and monitoring bats’ responses to climatic and land-cover changes. Ecological Indicators, 110(October 2019), 105849. https://doi.org/10.1016/j.ecolind.2019.105849

United States Naval Observatory. (2016). Sun or Moon Rise/Set Table for One Year. Retrieved August 30, 2019, from https://aa.usno.navy.mil/data/docs/RS OneYear.php

\section{Figure and Table Captions}

Figure 1: Study area on the Point Abbaye Peninsula in Baraga County, Michigan, USA. Points denote survey locations visited during April and May of 2019. The survey area covers $2.7 \mathrm{~km}^{2}$. White lines on the left panel show unploughed four wheel drive roads which are impassible from early April to early May each year.

Figure 2: The number of bird species detected by in-person point counts and automated recording units (ARUs) during the spring migration period on the Point Abbaye peninsula, Michigan, USA in 2019. Points show observed number of species detected, lines show predictions from a generalized linear mixed model, holding weather variables constant. See Box 1 for a description of the species richness index abbreviations. Listening to 10 random minutes of data from an ARU $\left(S_{10 \mathrm{R}}\right)$ allowed for detection of the same number of species as a 10 consecutive minute in-person point count $\left(S_{\mathrm{p}}\right)$. Increasing survey effort to 22 random minutes of ARU data $\left(S_{22 R}\right)$ increased the number of species detected to above the number of species detected by in-person point counts. 
Figure 3: Predicted (lines) and observed (points) relative abundance of (a) through (d) Winter Wren, and (e) through (h) Golden-crowned Kinglet, in April and May of 2019 on the Point Abbaye peninsula.

529 Points show observed values for each abundance index, while lines show the mean predicted values

530 from 200 five-fold cross validated boosted regression tree models. The vertical axes show the daily

531 abundance index value (see Box 1) calculated from: (a) and (e) three 10-minute in person point counts,

(b) and (f) three samples of 10 consecutive minutes of audio recordings from automated recording units (ARUs), (c) and (g) three samples of 10 randomly selected minutes of audio recordings from ARUs, (d) and (h) three samples of 22 randomly selected minutes of audio recordings from ARUs.

Figure 4: Correlation between daily observed values of relative abundance indices (Box 1) for Winter (the proportion of 30-second intervals with a vocalization) are correlated with the abundance index by abundance index; absolute values are less important here than the relationship between observations.

541 Photo: “Winter Wren” by ilouque, used under license CC BY 2.0. Cropped from original.

543 Figure 5: Correlation between daily observed values of relative abundance indices (Box 1) for Golden544 crowned Kinglet. See Table 2 for Spearman's correlation coefficients for each plot. Abundance indices

545 for ARUs (the proportion of 30-second intervals with a vocalization) are correlated with the abundance 546 index from point counts (mean number of individuals observed per count per day). Note that axis scales 547 vary by abundance index; absolute values are less important here than the relationship between 
548 observations. Photo: “Golden-crowned Kinglet” by Laura Gooch, used under license CC BY-NC-SA

549 2.0. Cropped from original.

550

551

Box 1: Indices of relative abundance and species richness for both in-person point count observations, and desk-based listening counts using audio data from Automated Recording Units (ARUs).

553

554 Table 1: Results of a generalized linear mixed model of the number of species detected as a function of 555 day of year, count type and environmental condition covariates. Each variable included in the model is 556 shown, along with the coefficient point estimate, 95\% confidence interval, and significance level.

$557 *$ denotes $\mathrm{p}<0.05$, ** denotes $\mathrm{p}<0.01$, *** denotes $\mathrm{p}<0.001$

559 Table 2: Spearman's rank correlation coefficients for each combination of abundance indices. We did

560 not report a correlation for $A_{30 R}$ and $A_{66 R}$ because $A_{30 R}$ data is a subset of $A_{66 R}$ data. 
bioRxiv preprint doi: https://doi.org/10.1101/2020.08.01.232215; this version posted August 3, 2020. The copyright holder for this preprint (which was not certified by peer review) is the author/funder, who has granted bioRxiv a license to display the preprint in perpetuity. It is made available under aCC-BY-ND 4.0 International license.

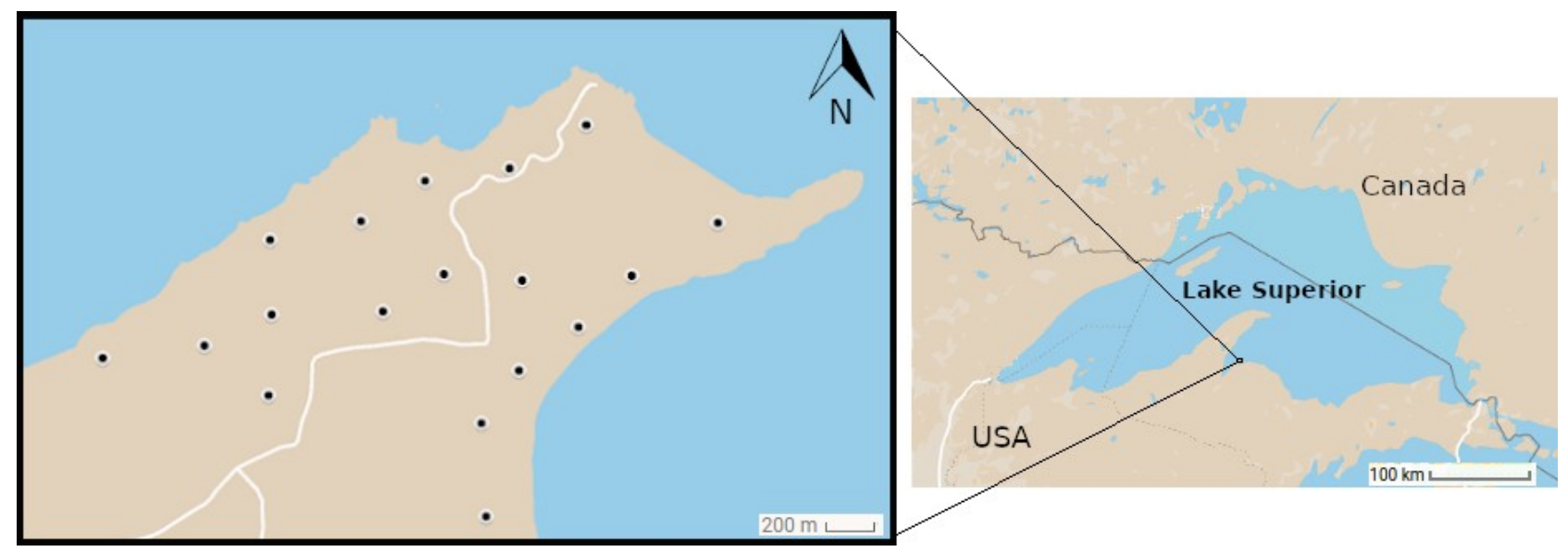

562

563

564

565

Figure 1

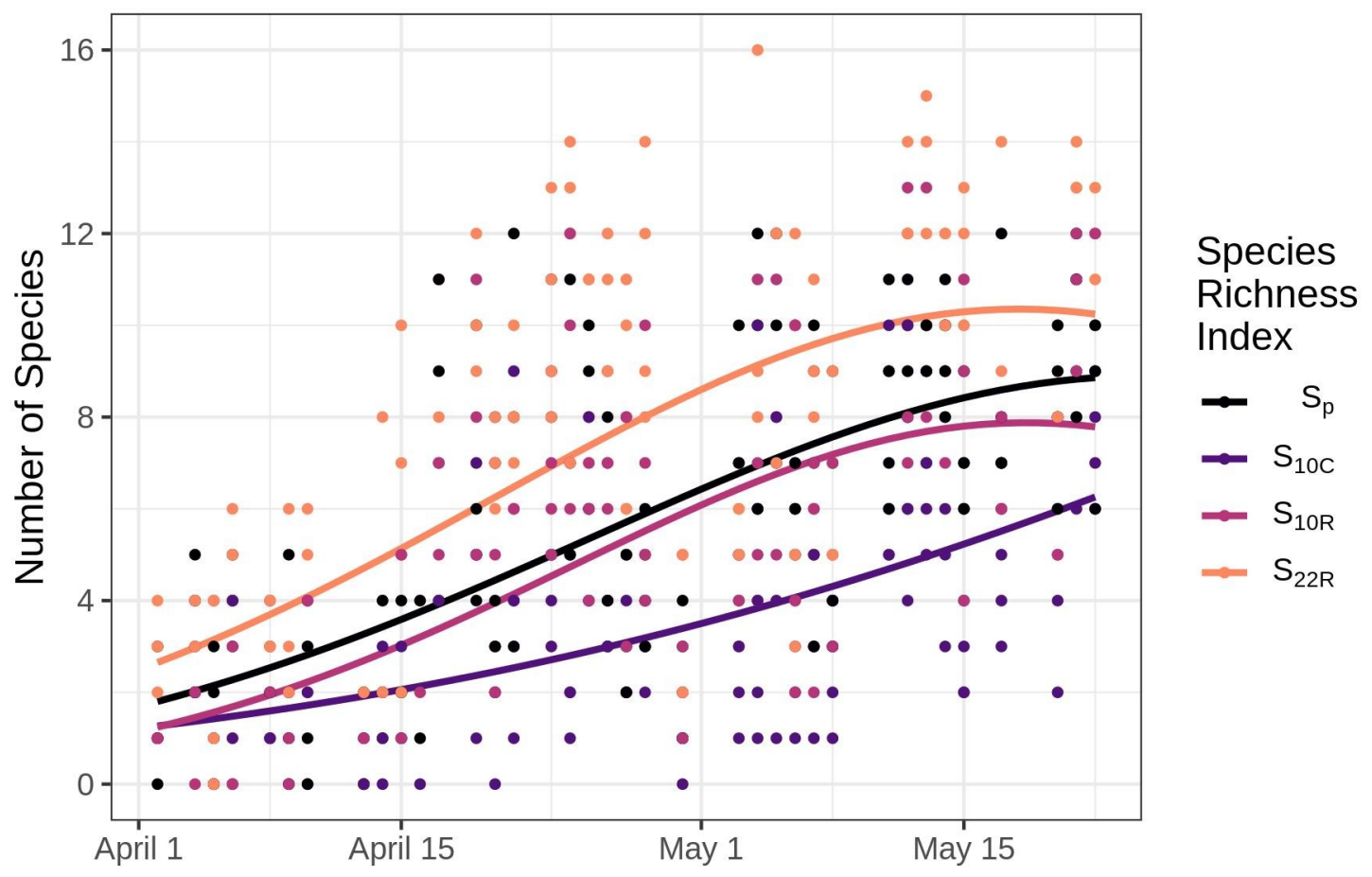

Figure 2 
(a)

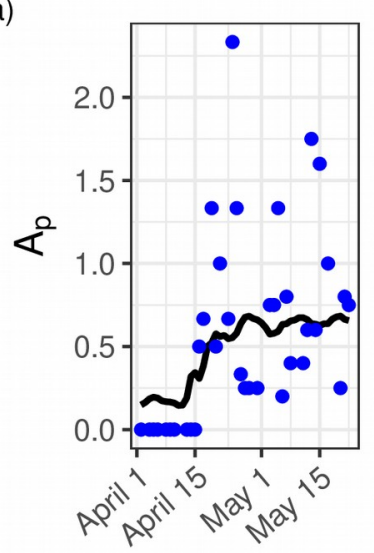

(e)

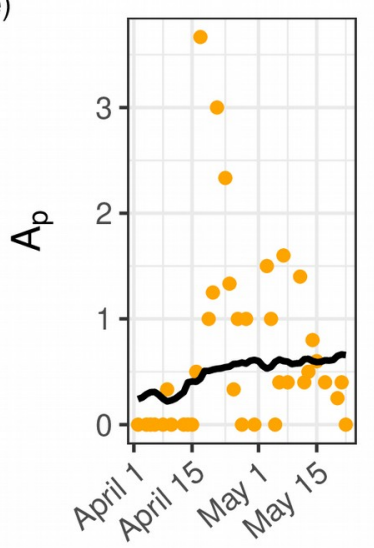

(b)

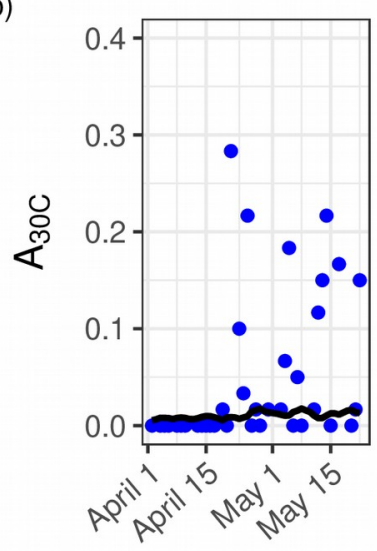

(f)

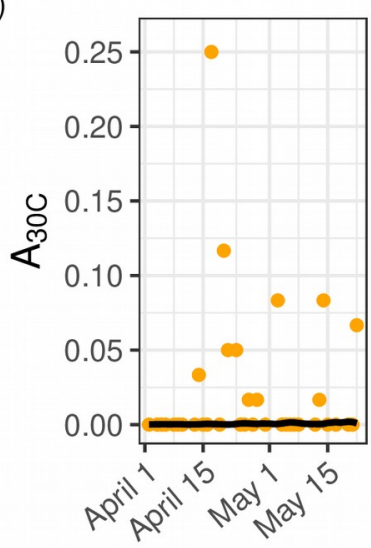

(c)

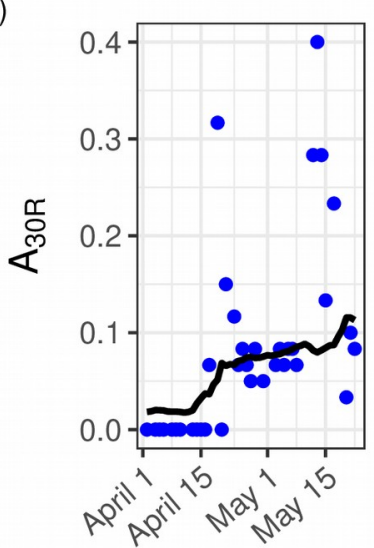

(g)

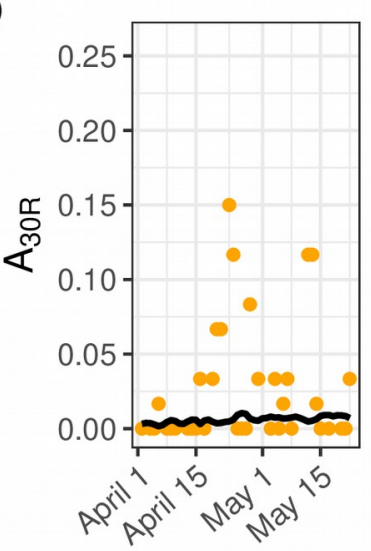

(d)

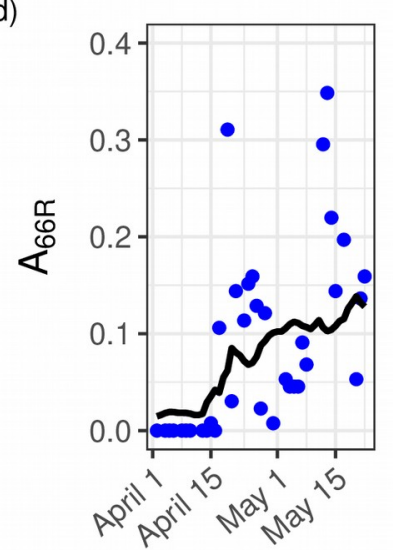

(h)

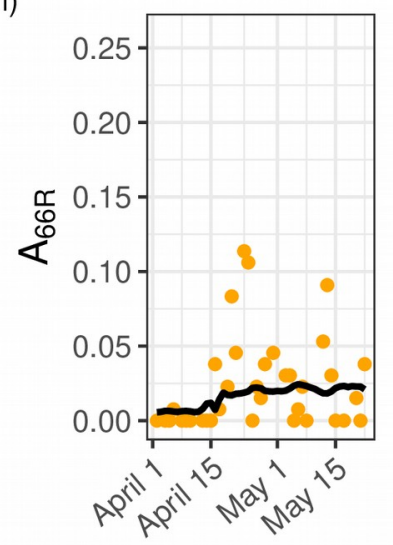


(a)
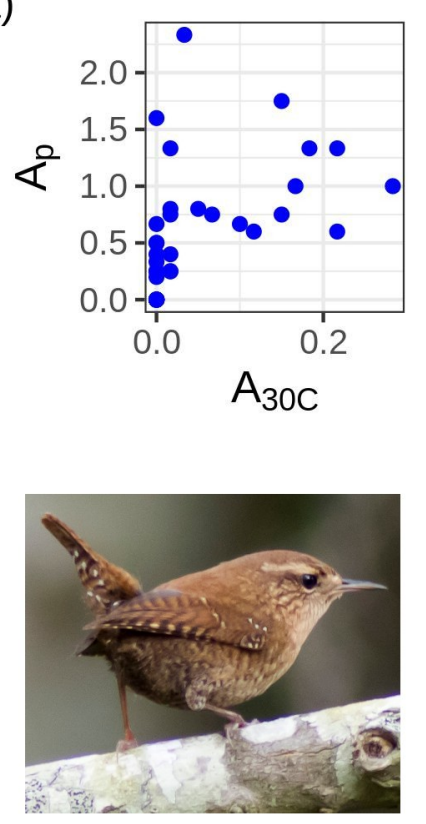

(b)

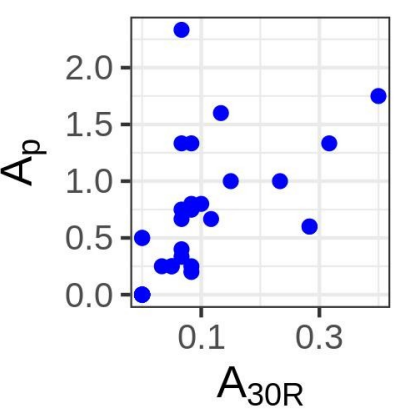

(d)

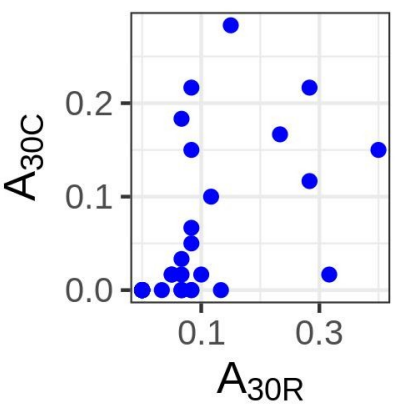

(c)

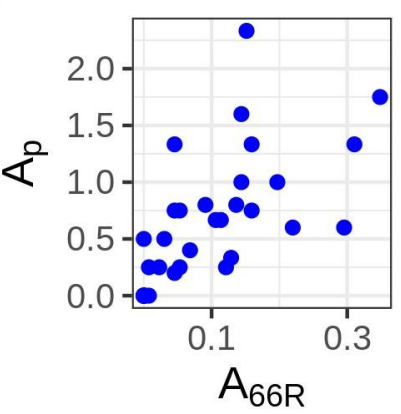

(e)

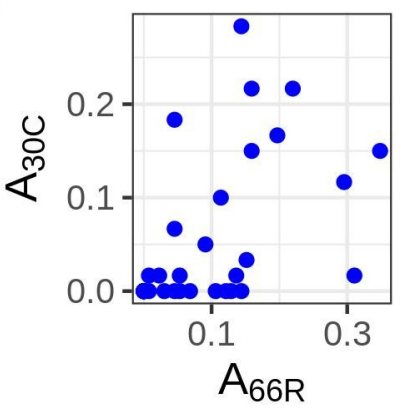



available under ACC-BY-ND 4.0 International license.

(a)
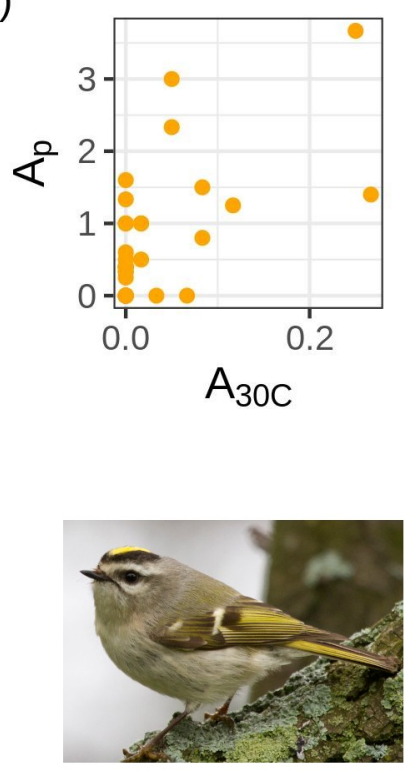

(b)

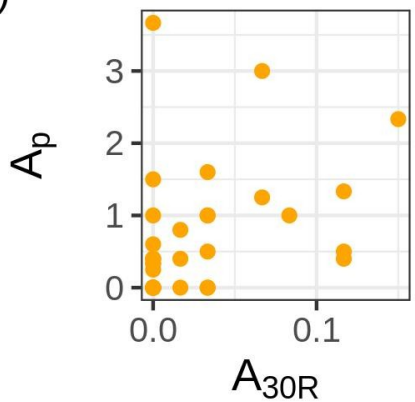

(d)

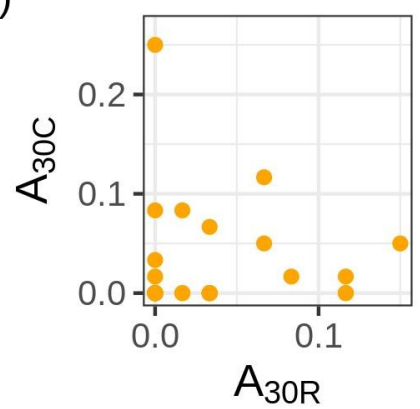

(c)

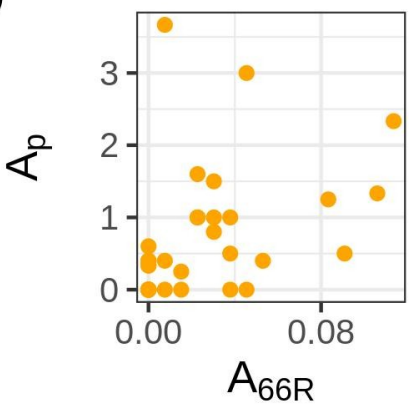

(e)

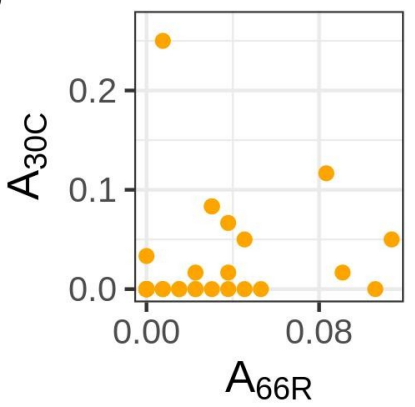

$574 \quad$ Figure 5 
Box 1

\begin{tabular}{|c|l|l|l|}
\hline $\begin{array}{l}\text { Index } \\
\text { Abbreviation }\end{array}$ & Survey type & Temporal continuity & Measured Value \\
\hline $\mathrm{A}_{\mathrm{p}}$ & Point count & Consecutive & $\begin{array}{l}\text { Relative abundance measured as the } \\
\text { mean number of observed } \\
\text { individuals per } 10 \text { minute point } \\
\text { count per day }\end{array}$ \\
\hline $\mathrm{A}_{n \mathrm{C}}$ & $\begin{array}{l}\text { Automated Recording } \\
\text { Unit }\end{array}$ & Consecutive & $\begin{array}{l}\text { Relative abundance measured as the } \\
\text { proportion of 30 second intervals } \\
\text { with a vocalization calculated by } \\
\text { surveying } n \text { minutes in sections of } \\
10 \text { consecutive minutes }\end{array}$ \\
\hline $\mathrm{A}_{n \mathrm{R}}$ & $\begin{array}{l}\text { Automated Recording } \\
\text { Unit }\end{array}$ & Random & $\begin{array}{l}\text { Relative abundance measured as the } \\
\text { proportion of 30 second intervals } \\
\text { with a vocalization calculated by } \\
\text { surveying } n \text { minutes in sections of } 1 \\
\text { minute chosen randomly from the } \\
\text { full five hour survey window. }\end{array}$ \\
\hline $\mathrm{S}_{n \mathrm{R}}$ & $\begin{array}{l}\text { Automated Recording } \\
\text { Unit }\end{array}$ & Random & $\begin{array}{l}\text { Number of species detected during } \\
\text { a 10 minute in person point count }\end{array}$ \\
\hline $\mathrm{S}_{n \mathrm{C}}$ & $\begin{array}{l}\text { Point count } \\
\text { Anit }\end{array}$ & Consecutive & $\begin{array}{l}\text { Number of species detected during } \\
n \text { consecutive minutes, in sections } \\
\text { of 10 consecutive minutes }\end{array}$ \\
\hline $\begin{array}{l}\text { Number of species detected during } \\
n \text { minutes, chosen randomly from } \\
\text { the full five hour survey window }\end{array}$ \\
\hline
\end{tabular}


Table 1

\begin{tabular}{|c|c|c|c|c|}
\hline Variable & $\begin{array}{c}\text { Coefficient } \\
\text { estimate }\end{array}$ & $\begin{array}{c}\text { 95\% CI } \\
\text { lower } \\
\text { bound }\end{array}$ & $\begin{array}{c}\text { 95\% CI } \\
\text { upper } \\
\text { bound }\end{array}$ & $P$-value \\
\hline Survey type $\left(\mathrm{S}_{10 \mathrm{C}}\right)$ & -0.6146 & -0.7824 & -0.4467 & $<0.0001 * * *$ \\
\hline Survey type $\left(\mathrm{S}_{10 \mathrm{R}}\right)$ & -0.065 & -0.213 & 0.0829 & 0.3888 \\
\hline Survey type $\left(\mathrm{S}_{22 \mathrm{R}}\right)$ & 0.3056 & 0.1702 & 0.441 & $<0.0001^{* * *}$ \\
\hline Wind (2) & -0.0301 & -0.135 & 0.0748 & 0.574 \\
\hline Wind $(3+)$ & -0.1007 & -0.2644 & 0.0629 & 0.2277 \\
\hline Rain (Wet) & -0.1984 & -0.4624 & 0.0657 & 0.1409 \\
\hline Noise (1) & 0.1552 & 0.0595 & 0.251 & $0.0015 * *$ \\
\hline Noise $(>2)$ & 0.0544 & -0.147 & 0.2557 & 0.5967 \\
\hline Day of year & 0.4346 & 0.305 & 0.5641 & $<0.0001^{* * *}$ \\
\hline Day of year² & -0.1159 & -0.2496 & 0.0178 & 0.0892 \\
\hline Survey type $\left(\mathrm{S}_{10 \mathrm{C}}\right)$ x Rain (Wet) & -0.0163 & -0.3944 & 0.3617 & 0.9325 \\
\hline Survey type $\left(\mathrm{S}_{10 \mathrm{R}}\right)$ x Rain (Wet) & 0.1861 & -0.1374 & 0.5096 & 0.2595 \\
\hline Survey type $\left(\mathrm{S}_{22 \mathrm{R}}\right)$ x Rain (Wet) & 0.2288 & -0.0677 & 0.5252 & 0.1304 \\
\hline Survey type $\left(\mathrm{S}_{10 \mathrm{c}}\right)$ x Day of Year & 0.0153 & -0.1191 & 0.1498 & 0.823 \\
\hline Survey type $\left(S_{10 R}\right)$ x Day of Year & 0.0595 & -0.0659 & 0.1849 & 0.3525 \\
\hline Survey type $\left(\mathrm{S}_{22 \mathrm{R}}\right)$ x Day of Year & -0.0709 & -0.1808 & 0.0389 & 0.2058 \\
\hline Survey type $\left(\mathrm{S}_{10 \mathrm{c}}\right)$ x Day of year ${ }^{2}$ & 0.086 & -0.048 & 0.2199 & 0.2083 \\
\hline Survey type $\left(\mathrm{S}_{10 \mathrm{R}}\right)$ x Day of year ${ }^{2}$ & -0.0583 & -0.1825 & 0.0659 & 0.3575 \\
\hline Survey type $\left(S_{22 R}\right) \times$ Day of year ${ }^{2}$ & -0.0148 & -0.125 & 0.0953 & 0.7917 \\
\hline
\end{tabular}




\section{Table 2}

\begin{tabular}{|c|c|c|c|c|c|}
\hline \multirow{2}{*}{\multicolumn{2}{|c|}{$\begin{array}{c}\text { Relative Abundance Index } \\
\text { Pairs }\end{array}$}} & \multicolumn{2}{|c|}{ Golden-crowned Kinglet } & \multicolumn{2}{|c|}{ Winter Wren } \\
\hline & & \multirow{2}{*}{\begin{tabular}{|c|c|}
$\begin{array}{c}\text { Correlation of } \\
\text { Observed } \\
\text { Values }\end{array}$ \\
0.532 \\
\end{tabular}} & \multirow{2}{*}{$\begin{array}{c}\begin{array}{c}\text { Correlation of } \\
\text { Predicted } \\
\text { Values }\end{array} \\
0.794\end{array}$} & \multirow{2}{*}{$\begin{array}{c}\begin{array}{c}\text { Correlation of } \\
\text { Observed } \\
\text { Values }\end{array} \\
0.72\end{array}$} & \multirow{2}{*}{\begin{tabular}{|c|c|}
$\begin{array}{c}\text { Correlation of } \\
\text { Predicted } \\
\text { Values }\end{array}$ \\
0.822 \\
\end{tabular}} \\
\hline$A_{P}$ & $\mathrm{~A}_{30 \mathrm{C}}$ & & & & \\
\hline$A_{P}$ & $\mathrm{~A}_{30 \mathrm{R}}$ & 0.499 & 0.652 & 0.781 & 0.882 \\
\hline$A_{P}$ & $\mathrm{~A}_{66 \mathrm{R}}$ & 0.588 & 0.813 & 0.817 & 0.854 \\
\hline $\mathrm{A}_{30 \mathrm{C}}$ & $\mathrm{A}_{30 \mathrm{R}}$ & 0.286 & 0.817 & 0.716 & 0.757 \\
\hline$A_{30 C}$ & $A_{66 R}$ & 0.485 & 0.897 & 0.676 & 0.736 \\
\hline
\end{tabular}

